\title{
Relationships Between the Cardio-Ankle Vascular Index and Pulsatility Index of the Common Carotid Artery in Patients With Cardiovascular Risk Factors
}

\author{
Takashi Hitsumoto
}

\begin{abstract}
Background: Pulsatility index (PI) is a hemodynamic parameter determined using Doppler sonography; it reflects the degree of peripheral vascular resistance. Moreover, researchers have reported significant relationships between an increase in the PI of the common carotid artery (CCA) and ischemic stroke. This cross-sectional study aimed to clarify the relationships between cardio-ankle vascular index (CAVI) as a marker of arterial stiffness and the PI of the CCA in patients with cardiovascular risk factors for the primary prevention of ischemic stroke.
\end{abstract}

Methods: A total of 405 outpatients undergoing treatment for cardiovascular risk factors (152 men and 253 women; mean age \pm standard deviation, $64 \pm 9$ years) with no history of cardiovascular events, including ischemic stroke, were enrolled. The CAVI and the PI of the CCA were measured using commercial devices, and their relationships to various clinical parameters were examined.

Results: A significant positive correlation was observed between the CAVI and the PI of the CCA $(r=0.44, P<0.001)$. Further, multiple regression analysis revealed that the CAVI $(\beta=0.19, \mathrm{P}<0.001)$ was selected as independent factor for PI of the CCA as a subordinate factor. Meanwhile, high-sensitivity C-reactive protein, as an inflammation marker, and skin autofluorescence, as a marker of advanced glycation end products in the tissues, were selected as independent variables for either the CAVI or the PI of the CCA as a subordinate factor. The receiver-operating characteristic curve analysis indicated that the cut-off point of the CAVI for high PI of the CCA $(>1.60)$ as a risk value of stroke incidence by previous report was 9.1 (area under the curve $=0.750, \mathrm{P}<0.001)$.

Conclusion: The present results indicate that the CAVI reflects cerebrovascular resistance in patients with cardiovascular risk factors. Moreover, the risk value of the CAVI for ischemic stroke incidence was considered to be 9.1 in these patients.

Keywords: Cardio-ankle vascular index; Pulsatility index; Common carotid artery; Inflammation; Skin autofluorescence; Cardiovascular

Manuscript submitted June 21, 2019, accepted July 13, 2019

Hitsumoto Medical Clinic, 2-7-7, Takezakicyou, Shimonoseki City, Yamaguchi 750-0025, Japan. Email: thitsu@jcom.home.ne.jp

doi: https://doi.org/10.14740/jocmr3914 risk factors

\section{Introduction}

Ischemic stroke is a common cardiovascular disease that is closely associated with the patient's quality of life. Moreover, many patients with cardiovascular risk factors, such as hypertension, type 2 diabetes mellitus and dyslipidemia, commonly experience ischemic stroke [1-3]. Therefore, it is important to consider effective methods to prevent ischemic stroke incidence in patients with cardiovascular risk factors.

Pulsatility index (PI) is a hemodynamic parameter determined using Doppler sonography and reflects the degree of peripheral vascular resistance. Clinical studies have reported that the PI of the carotid or cerebral artery is associated not only with arteriosclerosis of the cerebral vessels but also with the incidence of ischemic stroke [4-6]. During an ultrasonography examination of the carotid or the cerebral artery, the common carotid artery (CCA) can be easily detected in a clinical setting. Furthermore, several researchers have reported that an increase in the PI of the CCA reflects the risk of ischemic stroke $[5,6]$.

The cardio-ankle vascular index (CAVI) is a novel physiological marker of arteriosclerosis, reflecting arterial stiffness in the aorta, femoral and tibial arteries [7]. Recent clinical studies have emphasized the clinical importance of CAVI as a cardiovascular risk factor [8-10]. Moreover, several researchers have reported significant relationships between the CAVI and ischemic stroke $[8,11]$, suggesting that the CAVI reflects cerebrovascular hemodynamics. However, limited information is available regarding the clinical significance of the CAVI in cerebrovascular hemodynamics. This study examined the relationship between the CAVI and the PI of CCA in patients with cardiovascular risk factors for the primary prevention of ischemic stroke.

\section{Materials and Methods}

\section{Patients}

This cross-sectional study was conducted at the Hitsumoto 
Medical Clinic in Shimonoseki City from March 2016 to February 2019. The study population comprised 405 outpatients being treated for cardiovascular risk factors, such as hypertension, type 2 diabetes mellitus and dyslipidemia, who underwent an ultrasonographic examination of the carotid arteries. No patient had a history of cardiovascular diseases, such as stroke, coronary artery disease, or peripheral arterial disease. The patients comprised 152 men and 253 women with a mean age ( \pm standard deviation (SD)) of $64( \pm 9)$ years. The study was approved by the Institutional Review Board of the Hitsumoto Medical Clinic (approval number 2016-03) and was conducted in compliance with the Declaration of Helsinki.

\section{Measurement of the CAVI}

The CAVI was measured using a VaSera CAVI instrument (Fukuda Denshi, Tokyo, Japan) following the previously described methods [7]. Briefly, the brachial and ankle pulse waves were determined using inflatable cuffs with the pressure maintained between 30 and $50 \mathrm{~mm} \mathrm{Hg}$ to ensure minimal effect on the systemic hemodynamics. Systemic blood and pulse pressures were simultaneously determined, with the participant in the supine position. The CAVI was measured after the participants had rested for $10 \mathrm{~min}$ in a quiet room. For the statistical evaluation of the CAVI, mean values of the left and right sides were used. The CAVI may be less accurate in the presence of non-sinus rhythm; therefore, patients with chronic atrial fibrillation were excluded. The CAVI was calculated using the following formula: CAVI $=\mathrm{a}((2 \rho / \Delta \mathrm{P}) \times \ln (\mathrm{Ps} / \mathrm{Pd})$ PWV2) $+b$, where a and $b$ are constants, $\rho$ is blood density, $\Delta \mathrm{P}$ is $\mathrm{Ps}-\mathrm{Pd}$, Ps is systolic blood pressure, $\mathrm{Pd}$ is diastolic blood pressure and PWV is pulse wave velocity. The average coefficient of CAVI variation was shown to be $<5 \%$; this is small enough for clinical use and indicates that the CAVI measurement has good reproducibility [7].

\section{Ultrasonographic examination of the carotid artery}

Ultrasonographic examination of the carotid arteries was performed using a high-resolution ultrasonographic scanner with a 9-MHz linear array transducer (HI VISION Avius, Hitachi Medical Corporation, Tokyo, Japan). The PI of the CCA was measured as previously reported [5]. Briefly, the pulsed wave Doppler measurements were performed with the sample volume located in the middle CCA region with a maximum Doppler angle of $60^{\circ}$. Using a cine-loop function, the peak systolic velocity (PSV), end diastolic velocity (EDV) and time-averaged velocity (TAV) were calculated using software, and the PI was automatically calculated as follows: PI $=((\mathrm{PSV}-\mathrm{EDV}) /$ TAV). The average of the PI values of the right and left carotid arteries was calculated and defined as PI. The maximum intima-media thickness of all the carotid arteries was defined as max intima-media thickness (max-IMT), as previously reported [12]. In the present study, no patient had total occlusion of the CCA or the internal carotid artery.

\section{Evaluation of cardiovascular risk factors}

The degree of obesity was estimated using body mass index, calculated as weight in kilograms divided by height in meters squared. Current smoking was defined as smoking at least one cigarette per day during the previous 28 days. Hypertension was defined as systolic blood pressure $\geq 140 \mathrm{~mm} \mathrm{Hg}$, diastolic blood pressure $\geq 90 \mathrm{~mm} \mathrm{Hg}$, or the use of antihypertensive medication. Diabetes mellitus was defined as a fasting blood glucose level $\geq 126 \mathrm{mg} / \mathrm{dL}$, hemoglobin A1c (HbA1c) level $\geq$ $6.5 \%$, or the use of antidiabetic medication or exogenous insulin. Skin autofluorescence (AF) that reflects the accumulation of advanced glycation end products (AGEs), was measured on the volar side of the forearm using a commercial instrument (AGE Reader ${ }^{\mathrm{TM}}$; DiagnOptics, Groningen, The Netherlands), as previously described [13]. Dyslipidemia was defined as lowdensity lipoprotein cholesterol level $\geq 140 \mathrm{mg} / \mathrm{dL}$, high-density lipoprotein cholesterol level $\leq 40 \mathrm{mg} / \mathrm{dL}$, triglyceride level $\geq$ $150 \mathrm{mg} / \mathrm{dL}$, or the use of lipid-lowering medication. Blood samples were collected from the antecubital veins in the morning after $12 \mathrm{~h}$ of fasting. Glucose levels were measured using the glucose oxidase method. Total cholesterol and triglyceride concentrations were measured using standard enzymatic methods. High- and low-density lipoprotein cholesterol concentrations were measured using selective inhibition and Friedewald formula, respectively [14]. Participants with a serum triglyceride concentration of $\geq 400 \mathrm{mg} / \mathrm{dL}$ were excluded because this method is accurate below this concentration. The estimated glomerular filtration rate (eGFR) was calculated using the adjusted Modification of Diet in Renal Disease Study equation proposed by the working group of the Japanese Chronic Kidney Disease Initiative [15]. High-sensitivity C-reactive protein (hs-CRP) concentration, as a marker of inflammation, was measured using high-sensitivity, latex-enhanced immunonephelometrics.

\section{Statistical analyses}

Data were analyzed using the Stat View-J 5.0 (HULINKS, Tokyo, Japan) and MedCalc for Windows version 14.8.1 (MedCalc Software, Ostend, Belgium) and are expressed as mean \pm standard deviation values. The correlation coefficient was estimated using the Pearson or Spearman rank-order correlation analysis. Multivariate analysis was performed using multiple regression analyses. The receiver-operating characteristic curves were constructed, and the maximum Youden index [16] was used to determine the optimal CAVI cut-off levels of high PI of the CCA. A P-value $<0.05$ was considered statistically significant.

\section{Results}

\section{Study population}

Table 1 summarizes the patient characteristics. The mean PI of the CCA was $1.59 \pm 0.26$ (range, $1.10-2.82$ ), and the mean CAVI was $8.7 \pm 1.3$ (range, $6.3-13.6$ ). Both clinical param- 
Table 1. Patient Characteristics

\begin{tabular}{ll}
$\mathrm{n}$ (male/female) & $405(152 / 253)$ \\
\hline Age (years) & $64 \pm 9$ \\
Body mass index $\left(\mathrm{kg} / \mathrm{m}^{2}\right)$ & $22.7 \pm 3.7$ \\
Current smoker, $\mathrm{n}(\%)$ & $104(26)$ \\
Hypertension, $\mathrm{n}(\%)$ & $286(71)$ \\
Systolic blood pressure (mm Hg) & $139 \pm 11$ \\
Diastolic blood pressure (mm Hg) & $86 \pm 9$ \\
Diabetes mellitus, $\mathrm{n}(\%)$ & $169(42)$ \\
Fasting blood glucose (mg/dL) & $114 \pm 24$ \\
HbAlc (\%) & $6.2 \pm 1.1$ \\
Skin AF (AU) & $2.5 \pm 0.6$ \\
Dyslipidemia, $\mathrm{n}(\%)$ & $251(62)$ \\
Total cholesterol (mg/dL) & $212 \pm 41$ \\
LDL-cholesterol (mg/dL) & $134 \pm 37$ \\
Triglyceride (mg/dL) & $129 \pm 65$ \\
HDL-cholesterol (mg/dL) & $51 \pm 14$ \\
eGFR (mL/min/1.73 m² $)$ & $62 \pm 21$ \\
Log-hs-CRP (mg/dL) & $-1.2 \pm 0.7$ \\
PI of CCA & $1.59 \pm 0.26$ \\
Max-IMT (mm) & $1.36 \pm 0.49$ \\
CAVI & $8.7 \pm 1.3$ \\
Medication & $222(55)$ \\
$\quad$ RAS inhibitor, $\mathrm{n}(\%)$ & $167(41)$ \\
\hline \multicolumn{1}{c}{ Statin, $\mathrm{n}(\%)$} & $133(33)$ \\
\hline
\end{tabular}

Continuous values are mean $\pm \mathrm{SD}$. HbA1c: hemoglobinA1c; $\mathrm{FF}$ : autofluorescence; AU: arbitrary units; LDL: low-density lipoprotein; HDL: high-density lipoprotein; eGFR: estimated glomerular filtration rate; hs-CRP: high-sensitivity C-reactive protein; PI: pulsatility index; CCA: common carotid artery; IMT: intima-media thickness; CAVI: cardio-ankle vascular index; RAS: renin-angiotensin system.

eters had nearly normal distribution.

\section{Correlation between the factors}

The correlations between the PI of the CCA and the CAVI are shown in Figure 1. A significant positive correlation was observed between these two clinical parameters. Table 2 presents the relationship between the PI of the CCA, the CAVI and various clinical parameters. Age, smoking habits, presence of hypertension, systolic blood pressure, presence of diabetes mellitus, HbAlc, skin AF, eGFR, hs-CRP, max-IMT, renin angiotensin system inhibitor use, statin use and antidiabetic drugs use were significantly correlated with the PI of the CCA. In contrast, age, smoking habits, presence of hypertension, systolic blood pressure, diabetes mellitus related parameters, eGFR, hs-CRP, max-IMT, renin angiotensin system inhibitor and statin use were significantly correlated with the CAVI.

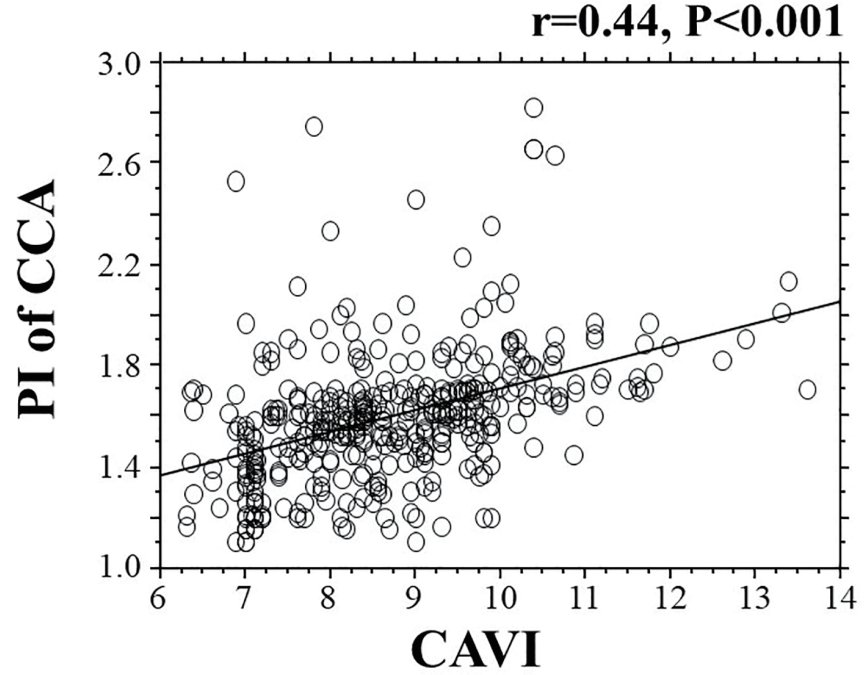

Figure 1. The correlation between the CAVI and the PI of the CCA. CAVI: cardio-ankle vascular index; PI: pulsatility index; CCA: common carotid artery.

\section{Multivariate analyses}

Table 3 summarizes the results of a multiple regression analyses with the PI of the CCA or the CAVI as subordinate factors. Explanatory factors were selected by examining the multicollinearity among the variables or by conducting a stepwise method. Skin AF, hs-CRP, CAVI, diabetes mellitus and maxIMT were selected as explanatory factors when the PI of the CCA was used as a subordinate factor. However, skin AF, max-IMT, PI of the CCA, hs-CRP and age were selected as explanatory factors when the CAVI was used as a subordinate factor. All the explanatory factors were selected as independent variables for the PI of the CCA or the CAVI as a subordinate factor.

\section{Receiver-operating characteristic curve analysis}

Figure 2 shows the receiver-operating characteristic curve analysis for the detection of high PI of the CCA as $>1.60$ based on previous report [6]. The maximum Youden index indicated that a CAVI of $>9.1$ was the optimal cut-off point to determine high PI (area under the curve $=0.750, \mathrm{P}<0.001$ ) with a true positive rate of $56.5 \%$ and a false positive rate of $82.6 \%$.

\section{Discussion}

This study aimed to clarify the relationships between the CAVI as a marker of arterial stiffness and the PI of the CCA in patients with cardiovascular risk factors for the primary prevention of ischemic stroke. Several studies have investigated the relationships between the CAVI and clinical parameters using carotid artery ultrasonography, and researchers have reported a significant association between the CAVI and IMT $[17,18]$. The 
Table 2. Relationship Between the PI of the CCA, the CAVI and Various Clinical Parameters

\begin{tabular}{|c|c|c|}
\hline & \multicolumn{2}{|c|}{$\mathbf{r}$} \\
\hline & PI of CCA & CAVI \\
\hline Sex $($ female $=0$, male $=1)$ & -0.01 & 0.07 \\
\hline Age & $0.28 * * *$ & $0.32 * * *$ \\
\hline Body mass index & -0.01 & -0.03 \\
\hline Current smoker $($ no $=0$, yes $=1)$ & $0.13^{*}$ & $0.13 * *$ \\
\hline Hypertension $($ no $=0$, yes $=1)$ & $0.10^{*}$ & $0.12 *$ \\
\hline Systolic blood pressure & $0.17 * *$ & $0.11^{*}$ \\
\hline Diastolic blood pressure & -0.06 & -0.08 \\
\hline Diabetes mellitus $($ no $=0$, yes $=1$ ) & $0.31 * * *$ & $0.14 * *$ \\
\hline Fasting blood glucose & 0.09 & $0.13 * *$ \\
\hline HbAlc & $0.16^{* *}$ & $0.19 * * *$ \\
\hline Skin AF & $0.38 * * *$ & $0.44 * * *$ \\
\hline Dyslipidemia $($ no $=0$, yes $=1)$ & -0.07 & -0.04 \\
\hline Total cholesterol & -0.02 & -0.06 \\
\hline LDL-cholesterol & -0.03 & -0.06 \\
\hline Triglyceride & 0.05 & 0.07 \\
\hline HDL-cholesterol & -0.03 & -0.05 \\
\hline eGFR & $-0.26 * * *$ & $-0.17 * * *$ \\
\hline Log-hs-CRP & $0.35^{* * *}$ & $0.33^{* * *}$ \\
\hline Max-IMT & $0.30 * * *$ & $0.34 * * *$ \\
\hline RAS inhibitor $($ no $=0$, yes $=1)$ & $-0.11 *$ & $-0.12^{*}$ \\
\hline Statin $($ no $=0$, yes $=1)$ & $-0.10^{*}$ & $-0.10^{*}$ \\
\hline Anti-diabetic drugs $($ no $=0$, yes $=1)$ & $0.21 * * *$ & 0.09 \\
\hline
\end{tabular}

$r$ expressed correlation coefficient. ${ }^{*} P<0.05$, ${ }^{* *} P<0.01$, ${ }^{* * *} P<0.001$. PI: pulsatility index; CCA: common carotid artery; CAVI: cardio-ankle vascular index; HbA1c: hemoglobinA1c; AF: autofluorescence; LDL: low-density lipoprotein; HDL: high-density lipoprotein; eGFR: estimated glomerular filtration rate; hs-CRP: high-sensitivity C-reactive protein; IMT: intima-media thickness; RAS: renin-angiotensin system.

results of this study also indicated that CAVI had independent association with max-IMT. In addition, there was independent association between CAVI and PI of CCA in this study, indicating that CAVI reflects not only carotid atherosclerosis but also cerebrovascular hemodynamics. On the contrary, hs-CRP and skin AF were selected as independent variables for either CAVI or PI of CCA as a subordinate factor.

The measurement theory of the PI is considered to reflect vascular resistance that is far distal from the point of examination. Therefore, all areas of the intracranial arterial dysfunction or stenosis can affect the PI of the CCA. However, Saji et al reported that the CAVI was significantly higher in patients with large infarcts attributed to large artery atherosclerosis or subcortical infarcts attributed to small-artery disease than in those without a history of stroke [19]. Thus, the results of this and Saji's study suggest that the CAVI reflects intracranial arterial dysfunction or stenosis not only in the large artery, but also in small sized vessels; consequently, patients with
Table 3. Multiple Regression Analyses

\begin{tabular}{|lll}
\hline Explanatory factor & $\boldsymbol{\beta}$ & P value \\
\hline (A) Skin AF & 0.25 & $<0.001$ \\
Log-hs-CRP & 0.20 & $<0.001$ \\
CAVI & 0.19 & $<0.001$ \\
Diabetes mellitus & 0.15 & 0.003 \\
Max-IMT & 0.14 & 0.009 \\
(B) Skin AF & 0.27 & $<0.001$ \\
Max-IMT & 0.19 & $<0.001$ \\
PI of CCA & 0.17 & 0.002 \\
Log-hs-CRP & 0.16 & 0.003 \\
Age & 0.15 & 0.007 \\
\hline
\end{tabular}

(A) Subordinate factor is PI of CCA; $R^{2}=0.37$; $<0.001$. (B) Subordinate factor is CAVI; $R^{2}=0.36 ; P<0.001$. AF: autofluorescence; hsCRP: high-sensitivity C-reactive protein; CAVI: cardio-ankle vascular index; IMT: intima-media thickness; PI: pulsatility index; CCA: common carotid artery; $\beta$ : standardized regression coefficient; $R^{2}$ : coefficient of determination.

high CAVI are at a higher risk of ischemic stroke. In contrast, other researchers have indicated that arterial stiffness caused cerebral small sized vessel disease $[20,21]$. Van Sloten et al indicated that increase in the pulsatile pressure and flow might directly lead to cerebral microcirculatory damage and induce a microvascular remodeling response [20]. Saji et al also reported that arterial stiffness is independently associated with

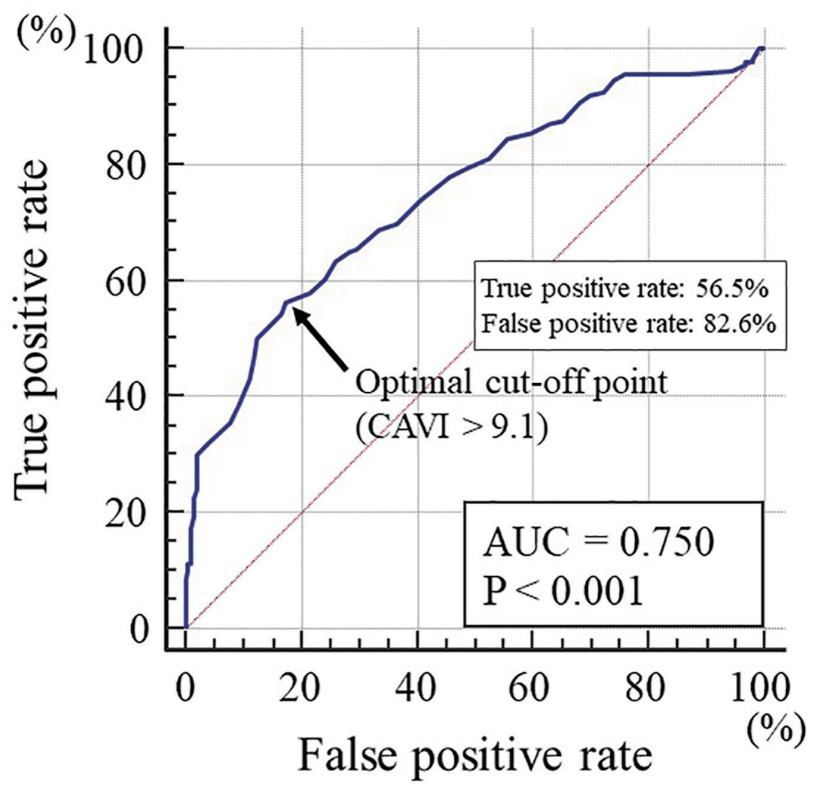

Figure 2. The receiver-operating characteristic curve analysis for the detection of high PI of the CCA based on the CAVI. The maximum Youden index indicated that a CAVI of $>9.1$ was the optimal cut-off point to determine high $\mathrm{PI}(\mathrm{AUC}=0.750, \mathrm{P}<0.001)$ with a true positive rate of $56.5 \%$ and a false positive rate of $82.6 \%$. PI: pulsatility index; CCA: common carotid artery; CAVI: cardio-ankle vascular index; AUC: area under the curve. 
all the components of cerebral small vessel disease, including silent lacunar infarcts, white matter hyperintensities and microbleeds [21]. Thus, the independent association between the CAVI and the PI of the CCA in this study can be partly explained by the cerebral small vessel injury caused by increased arterial stiffness.

Researchers emphasize that low-grade inflammation in the arterial vessel wall plays a crucial role in arterial dysfunction $[22,23]$. In fact, clinical studies have reported significant associations between inflammatory markers and the CAVI or ischemic stroke [24-26]. This study also indicated that hs-CRP was selected as an independent variable for both CAVI and PI of the CCA as subordinate factors, indicating that inflammation plays an important role in promoting systemic arterial dysfunction in patients with cardiovascular risk factors. However, medications, such as angiotensin receptor blockers or statins, were reported to decrease inflammation in the arterial wall [27, 28]. In this study, angiotensin receptor blockers or statins also had a significant negative correlation with the PI of the CCA or the CAVI in univariate analysis, although these medications were not selected in the multivariate model. Therefore, these medications, that is, angiotensin receptor blockers or statins, were aggressively administered to patients with high hs-CRP levels, consequently, possibly decreasing cardiovascular events, such as ischemic stroke, by decreasing the inflammation in the arterial vessel wall.

Recent basic and clinical studies have indicated a crucial role of AGEs or receptor of AGEs for arterial aging [2931]. Researchers have also reported the clinical usefulness of skin AF as a cardiovascular risk factor [32-34]. The present results also indicate an independent association of skin AF and PI of the CCA or the CAVI, suggesting that the accumulation of AGEs in the arterial vessel wall contributes to systemic arterial dysfunction, including that of the cerebral vessels in patients with cardiovascular risk factors. Genevieve et al examined the relationship between skin AF and $\mathrm{HbAlc}$ levels in patients with diabetes mellitus [35]. They measured the HbA1c levels every 6 months and found that the skin AF level was significantly related to the preceding five and ten mean HbA1c levels. However, other researchers have reported that lifestyle habits, such as physical activity, smoking status, sleep condition, mental stress level, sugary foods intake and intake of meat products were significantly associated with skin AF levels [36-38]. Thus, long-term glucose control in patients with diabetes mellitus and performance of good lifestyle habits are important to maintain lower AGEs levels and arterial function.

It is useful to know the target cut-off level of the CAVI for predicting abnormal PI of the CCA in clinical settings. This study clarified the clinical usefulness of assessing the CAVI for detecting high PI of the CCA of $>1.60$, demonstrated as a predictor of stroke events [6]. The receiver-operating characteristic curve analysis indicated that a CAVI of $>9.1$ was the optimal cut-off point for discriminating high PI of the CCA. In contrast, several studies have reported that a CAVI as high as $\geq 9.0$ is a risk factor for cardiovascular events [39, 40]. This study also suggests that the ischemic stroke can be prevented in patients with cardiovascular risk factors by maintaining the CAVI at approximately $\leq 9$ from the perspective of cerebro- vascular hemodynamics.

\section{Limitations}

This study has certain limitations. First, the medical treatments for cardiovascular risk factors, such as hypertension, diabetes mellitus and dyslipidemia, might have influenced the study results. Second, computed tomography and magnetic resonance imaging for brain were not completely performed for all patients. Therefore, cases of asymptomatic ischemic stroke may have remained undetected. Finally, this was a single-center cross-sectional study with a relatively small population. A prospective study involving a larger number of patients is necessary to confirm the validity of the present findings.

\section{Conclusions}

In sum, this study indicated an independent association between the CAVI and the PI of the CCA, suggesting that the CAVI reflects cerebrovascular resistance in patients with cardiovascular risk factors. Moreover, the risk value of the CAVI for the incidence of ischemic stroke was considered as 9.1 in these patients.

\section{Acknowledgments}

The author is grateful to the individuals who participated in this study.

\section{Financial Disclosure}

None to declare.

\section{Conflict of Interest}

None to declare.

\section{Informed Consent}

All patients provided informed consent.

\section{Author Contributions}

The author was involved in preparing the study design as well as in the acquisition, analysis and interpretation of data.

\section{References}

1. Huangfu X, Zhu Z, Zhong C, Bu X, Zhou Y, Tian Y, Batu $\mathrm{B}$, et al. Smoking, hypertension, and their combined ef- 
fect on ischemic stroke incidence: a prospective study among Inner Mongolians in China. J Stroke Cerebrovasc Dis. 2017;26(12):2749-2754.

2. Saito I. Epidemiological evidence of type 2 diabetes mellitus, metabolic syndrome, and cardiovascular disease in Japan. Circ J. 2012;76(5):1066-1073.

3. Tziomalos K, Athyros VG, Karagiannis A, Mikhailidis DP. Dyslipidemia as a risk factor for ischemic stroke. Curr Top Med Chem. 2009;9(14):1291-1297.

4. Lee KY, Sohn YH, Baik JS, Kim GW, Kim JS. Arterial pulsatility as an index of cerebral microangiopathy in diabetes. Stroke. 2000;31(5):1111-1115.

5. Nakatou T, Nakata K, Nakamura A, Itoshima T. Carotid haemodynamic parameters as risk factors for cerebral infarction in Type 2 diabetic patients. Diabet Med. 2004;21(3):223-229.

6. Chuang SY, Cheng HM, Bai CH, Yeh WT, Chen JR, Pan WH. Blood pressure, carotid flow pulsatility, and the risk of stroke: a community-based study. Stroke. 2016;47(9):22622268.

7. Shirai K, Utino J, Otsuka K, Takata M. A novel blood pressure-independent arterial wall stiffness parameter; cardio-ankle vascular index (CAVI). J Atheroscler Thromb. 2006;13(2):101-107.

8. Gohbara M, Iwahashi N, Sano Y, Akiyama E, Maejima N, Tsukahara K, Hibi K, et al. Clinical impact of the cardioankle vascular index for predicting cardiovascular events after acute coronary syndrome. Circ J. 2016;80(6):14201426.

9. Matsushita K, Ding N, Kim ED, Budoff M, Chirinos JA, Fernhall B, Hamburg NM, et al. Cardio-ankle vascular index and cardiovascular disease: Systematic review and meta-analysis of prospective and cross-sectional studies. J Clin Hypertens (Greenwich). 2019;21(1):16-24.

10. Hitsumoto T. Clinical Usefulness of the Cardio-Ankle Vascular Index as a Predictor of Primary Cardiovascular Events in Patients With Chronic Kidney Disease. J Clin Med Res. 2018;10(12):883-890.

11. Suzuki J, Sakakibara R, Tomaru T, Tateno F, Kishi M, Ogawa E, Kurosu T, et al. Stroke and cardio-ankle vascular stiffness index. J Stroke Cerebrovasc Dis. 2013;22(2):171-175.

12. Kasami R, Kaneto H, Katakami N, Sumitsuji S, Yamasaki K, Kuroda T, Tachibana K, et al. Relationship between carotid intima-media thickness and the presence and extent of coronary stenosis in type 2 diabetic patients with carotid atherosclerosis but without history of coronary artery disease. Diabetes Care. 2011;34(2):468-470.

13. Meerwaldt R, Links TP, Graaff R, Hoogenberg $\mathrm{K}$, Lefrandt JD, Baynes JW, Gans RO, et al. Increased accumulation of skin advanced glycation end-products precedes and correlates with clinical manifestation of diabetic neuropathy. Diabetologia. 2005;48(8):1637-1644.

14. Friedewald WT, Levy RI, Fredrickson DS. Estimation of the concentration of low-density lipoprotein cholesterol in plasma, without use of the preparative ultracentrifuge. Clin Chem. 1972;18(6):499-502.

15. Imai E, Horio M, Nitta K, Yamagata K, Iseki K, Hara $\mathrm{S}$, Ura N, et al. Estimation of glomerular filtration rate by the MDRD study equation modified for Japanese patients with chronic kidney disease. Clin Exp Nephrol. 2007;11(1):41-50.

16. Schisterman EF, Perkins NJ, Liu A, Bondell H. Optimal cut-point and its corresponding Youden Index to discriminate individuals using pooled blood samples. Epidemiology. 2005;16(1):73-81.

17. Takaki A, Ogawa H, Wakeyama T, Iwami T, Kimura M, Hadano Y, Matsuda S, et al. Cardio-ankle vascular index is superior to brachial-ankle pulse wave velocity as an index of arterial stiffness. Hypertens Res. 2008;31(7):13471355.

18. Hayashida H, Saito T, Kawasaki K, Kitamura M, Furugen $\mathrm{R}$, Iwasaki $\mathrm{T}$, Hayashida $\mathrm{Y}$, et al. Association of periodontitis with carotid artery intima-media thickness and arterial stiffness in community-dwelling people in Japan: the Nagasaki Islands study. Atherosclerosis. 2013;229(1):186-191.

19. Saji N, Kimura K, Yagita Y, Kawarai T, Shimizu H, Kita Y. Comparison of arteriosclerotic indicators in patients with ischemic stroke: ankle-brachial index, brachial-ankle pulse wave velocity and cardio-ankle vascular index. Hypertens Res. 2015;38(5):323-328.

20. van Sloten TT, Protogerou AD, Henry RM, Schram MT, Launer LJ, Stehouwer CD. Association between arterial stiffness, cerebral small vessel disease and cognitive impairment: A systematic review and meta-analysis. Neurosci Biobehav Rev. 2015;53:121-130.

21. Saji N, Toba K, Sakurai T. Cerebral Small Vessel Disease and Arterial Stiffness: Tsunami Effect in the Brain? Pulse (Basel). 2016;3(3-4):182-189.

22. Carbone F, Montecucco F. Inflammation in arterial diseases. IUBMB Life. 2015;67(1):18-28.

23. Della Corte V, Tuttolomondo A, Pecoraro R, Di Raimondo D, Vassallo V, Pinto A. Inflammation, Endothelial Dysfunction and Arterial Stiffness as Therapeutic Targets in Cardiovascular Medicine. Curr Pharm Des. 2016;22(30):4658-4668.

24. Kotani K, Yamada T, Miyamoto M, Kario K, Ishibashi $\mathrm{S}$, Taniguchi N. The correlation between the cardio-ankle vascular index (CAVI) and serum amyloid A in asymptomatic Japanese subjects. Heart Vessels. 2012;27(5):499504.

25. Higashiyama A, Wakabayashi I, Kubota Y, Adachi Y, Hayashibe A, Nishimura K, Sugiyama D, et al. Does highsensitivity C-reactive protein or low-density lipoprotein cholesterol show a stronger relationship with the cardioankle vascular index in healthy community dwellers?: the KOBE study. J Atheroscler Thromb. 2012;19(11):10271034.

26. Jin R, Liu L, Zhang S, Nanda A, Li G. Role of inflammation and its mediators in acute ischemic stroke. J Cardiovasc Transl Res. 2013;6(5):834-851.

27. Sifi A, Adi-Bessalem S, Laraba-Djebari F. Role of angiotensin II and angiotensin type-1 receptor in scorpion venom-induced cardiac and aortic tissue inflammation. Exp Mol Pathol. 2017;102(1):32-40.

28. van der Valk FM, Bernelot Moens SJ, Verweij SL, Strang AC, Nederveen AJ, Verberne HJ, Nurmohamed MT, et 
al. Increased arterial wall inflammation in patients with ankylosing spondylitis is reduced by statin therapy. Ann Rheum Dis. 2016;75(10):1848-1851.

29. Yan SF, Ramasamy R, Schmidt AM. The RAGE axis: a fundamental mechanism signaling danger to the vulnerable vasculature. Circ Res. 2010;106(5):842-853.

30. Suga T, Iso T, Shimizu T, Tanaka T, Yamagishi S, Takeuchi M, Imaizumi T, et al. Activation of receptor for advanced glycation end products induces osteogenic differentiation of vascular smooth muscle cells. J Atheroscler Thromb. 2011;18(8):670-683.

31. Grossin N, Auger F, Niquet-Leridon C, Durieux N, Montaigne D, Schmidt AM, Susen S, et al. Dietary CMLenriched protein induces functional arterial aging in a RAGE-dependent manner in mice. Mol Nutr Food Res. 2015;59(5):927-938.

32. Furuya F, Shimura H, Takahashi K, Akiyama D, Motosugi A, Ikegishi Y, Haraguchi K, et al. Skin autofluorescence is a predictor of cardiovascular disease in chronic kidney disease patients. Ther Apher Dial. 2015;19(1):40-44.

33. Noordzij MJ, Mulder DJ, Oomen PH, Brouwer T, Jager J, Castro Cabezas M, Lefrandt JD, et al. Skin autofluorescence and risk of micro- and macrovascular complications in patients with Type 2 diabetes mellitus-a multicentre study. Diabet Med. 2012;29(12):1556-1561.

34. Hitsumoto T. Clinical impact of skin autofluorescence on high-sensitivity troponin $\mathrm{T}$ in hypertensive patients. Clin Hypertens. 2017;23:19.
35. Genevieve M, Vivot A, Gonzalez C, Raffaitin C, Barberger-Gateau P, Gin H, Rigalleau V. Skin autofluorescence is associated with past glycaemic control and complications in type 1 diabetes mellitus. Diabetes Metab. 2013;39(4):349-354.

36. Isami F, West BJ, Nakajima S, Yamagishi SI. Association of advanced glycation end products, evaluated by skin autofluorescence, with lifestyle habits in a general Japanese population. J Int Med Res. 2018;46(3):1043-1051.

37. Botros N, Sluik D, van Waateringe RP, de Vries JHM, Geelen A, Feskens EJM. Advanced glycation endproducts (AGEs) and associations with cardio-metabolic, lifestyle, and dietary factors in a general population: the NQplus study. Diabetes Metab Res Rev. 2017;33(5):e2892.

38. Kellow NJ, Coughlan MT, Reid CM. Association between habitual dietary and lifestyle behaviours and skin autofluorescence (SAF), a marker of tissue accumulation of advanced glycation endproducts (AGEs), in healthy adults. Eur J Nutr. 2018;57(6):2209-2216.

39. Izuhara M, Shioji K, Kadota S, Baba O, Takeuchi Y, Uegaito T, Mutsuo S, et al. Relationship of cardio-ankle vascular index (CAVI) to carotid and coronary arteriosclerosis. Circ J. 2008;72(11):1762-1767.

40. Tanaka A, Tomiyama H, Maruhashi T, Matsuzawa Y, Miyoshi T, Kabutoya T, Kario K, et al. Physiological diagnostic criteria for vascular failure. Hypertension. 2018;72(5):1060-1071. 